\title{
Diagnosis of acute myeloid leukemia according to the WHO classification in the Japan Adult Leukemia Study Group AML-97 protocol
}

\author{
Moe Wakui - Kazutaka Kuriyama - Yasushi Miyazaki - Tomoko Hata • \\ Masafumi Taniwaki - Shigeki Ohtake · Hisashi Sakamaki · Shuichi Miyawaki · \\ Tomoki Naoe $\cdot$ Ryuzo Ohno $\cdot$ Masao Tomonaga
}

Received: 12 September 2007/Revised: 30 October 2007/Accepted: 2 November 2007/Published online: 7 February 2008

(C) The Japanese Society of Hematology 2008

\begin{abstract}
We reviewed and categorized 638 of 809 patients who were registered in the Japan Adult Leukemia Study Group acute myeloid leukemia (AML)-97 protocol using morphological means. Patients with the M3 subtype were excluded from the study group. According to the WHO classification, 171 patients (26.8\%) had AML with
\end{abstract}

\author{
M. Wakui · K. Kuriyama ( $₫)$ \\ Department of Clinical Laboratory Sciences, \\ Hematoimmunology, School of Health Science, \\ Faculty of Medicine, University of the Ryukyus, 207 Uehara, \\ Nishihara-cho, Okinawa 903-0215, Japan \\ e-mail: kuriyama@med.u-ryukyu.ac.jp \\ Y. Miyazaki - T. Hata $\cdot$ M. Tomonaga \\ Department of Hematology, Atomic Bomb Disease Institute, \\ Nagasaki University School of Medicine, Nagasaki, Japan \\ M. Taniwaki \\ Department of Hematology and Oncology, Kyoto Prefectural \\ University of Medicine, Kyoto, Japan \\ S. Ohtake \\ Department of Clinical Laboratory Science, \\ Kanazawa University Graduate School of Medical Science, \\ Kanazawa, Japan \\ H. Sakamaki \\ Department of Hematology, Tokyo Metropolitan Komagome \\ Hospital, Tokyo, Japan

\section{S. Miyawaki} \\ Department of Hematology, Saiseikai Maebashi Hospital, \\ Maebashi, Japan \\ T. Naoe \\ Department of Hematology and Oncology, Nagoya University \\ Graduate School of Medicine, Nagoya, Japan \\ R. Ohno \\ Aichi Cancer Center, Nagoya, Aichi, Japan
}

recurrent genetic abnormalities, 133 (20.8\%) had AML with multilineage dysplasia (MLD), 331 (51.9\%) had AML not otherwise categorized, and $3(0.5 \%)$ had acute leukemia of ambiguous lineage. The platelet count was higher and the rate of myeloperoxidase (MPO)-positive blasts was lower in AML with MLD than in the other WHO categories. The outcome was significantly better in patients with high $(\geq 50 \%)$ than with low $(<50 \%)$ ratios of MPO-positive blasts $(P<0.01)$. The 5 -year survival rates for patients with favorable, intermediate, and adverse karyotypes were 63.4, 39.1, and $0.0 \%$, respectively, and $35.5 \%$ for those with $11 \mathrm{q} 23$ abnormalities $(P<0.0001)$. Overall survival (OS) did not significantly differ between nine patients with $t(9 ; 11)$ and 23 with other 11q23 abnormalities $(P=0.22)$. Our results confirmed that the cytogenetic profile, MLD phenotype, and MPO-positivity of blasts are associated with survival in patients with AML, and showed that each category had the characteristics of the WHO classification such as incidence, clinical features, and OS.

Keywords AML - WHO classification . Myeloperoxidase $\cdot$ Multilineage dyplasia . $11 \mathrm{q} 23$ abnormalities

\section{Introduction}

The French-American-British (FAB) classification of acute myeloid leukemia (AML), based on morphological and cytochemical findings, was established in 1976 and has since become the standard classification $[1,2]$. However, specific chromosomal and genetic abnormalities that have been extracted from analyses of prognostic factors for AML are recognized as important in selecting treatment strategies and are reflected in the AML classification as 
factors that are required to establish the disease entity [3]. The 1999 World Health Organization (WHO) classification includes morphological, immunological, cytogenetic, genetic, and clinical features [4-6]. The WHO and FAB classifications differ in several aspects. The blast threshold required for a diagnosis of AML was reduced from 30 to $20 \%$, and new AML categories have been added for cytogenetic abnormalities, the presence of multilineage dysplasia (MLD), as well as a history of chemotherapy and subtypes for acute basophilic leukemia, acute panmyelosis with myelofibrosis, and myeloid sarcoma. The WHO classification comprises more subtypes and is more comprehensive than the FAB classification.

Cytogenetic features are important prognostic factors in AML [3, 7-12]. However, 11q23 abnormalities have not yet been established as a cytogenetic risk classification. Over 30 partner genes with 11q23 abnormalities have been described, and some reports indicate that patients with $t(9 ; 11)$ have a relatively more favorable prognosis than those with other partner chromosomes/partner genes [13-16].

In the present study, we reviewed stained smears of blood and bone marrow from patients who were registered in the Japan Adult Leukemia Study Group (JALSG) AML97 trial, and classified them into FAB subtypes and WHO categories. We also evaluated their survival on the basis of the WHO classification, the myeloperoxidase (MPO)-positivity of blasts, and cytogenetic findings including 11q23 abnormalities.

\section{Patients and methods}

\subsection{Patients}

Between December 1997 and July 2001, 809 patients aged from 15 to 66 years with untreated AML (excluding M3) were registered from 103 institutions in the AML-97 trial of the JALSG. The patients were diagnosed with AML according to the FAB criteria at each institution. Patients with a history of MDS, hematological abnormalities before the diagnosis of AML, or a history of chemotherapy were not eligible for the AML-97 trial.

\subsection{Treatment strategies}

Details of the JALSG AML-97 treatment protocol are described elsewhere [17]. In brief, all patients underwent induction therapy consisting of idarubicin (3 days) and AraC (7 days). Patients who achieved complete remission were randomized into one of two arms of consolidation chemotherapy alone or in combination with maintenance chemotherapy. Patients who were placed into intermediate/ poor risk groups according to the JALSG scoring system [17] and who had an HLA-identical sibling ( $\leq 50$ years old) were simultaneously assigned to receive allogeneic hematopoietic stem cell transplantation during their first remission.

\subsection{Morphologic and cytochemical analyses}

Peripheral blood and bone marrow smears from registered patients were sent to Nagasaki University for staining with May-Giemsa, MPO, and esterase, and the diagnosis was then reevaluated by the Central Review Committee for Morphological Diagnosis. Patients were subsequently categorized according to the FAB and WHO classifications. Dyserythropoietic features were defined as $>50 \%$ dysplastic features in at least 25 erythroblasts and dysgranulopoietic features including $\geq 3$ neutrophils with hyposegmented nuclei (pseudo-Pelger-Heut anomaly), and hypogranular or agranular neutrophils $(>50 \%$ of $\geq 10$ neutrophils). Dysmegakaryopoietic features were defined as $\geq 3$ megakaryocytes that were micronuclear, multiseparate nuclear, or large mononuclear [18].

We assessed the ratios (\%) of MPO-positive blasts on MPO-stained bone marrow smears using the diaminobenzidine method [19].

\subsection{Cytogenetic analysis}

Cytogenetic analysis was performed at either laboratories in participating hospitals or authorized commercial laboratories. The karyotypes of leukemic cells were collected through the JALSG AML-97 case report forms and reviewed by the Central Review Committee for Karyotyping. The patients were classified into favorable, intermediate, or adverse risk groups based on karyotypes according to results of the Medical Research Council (MRC) AML 10 trial [3]. The favorable risk group included patients with $t(8 ; 21)$ and inv(16), whether alone or in combination with other abnormalities. The intermediate risk group included those with a normal karyotype and other abnormalities that were not classified as either favorable or adverse. The adverse risk group included patients with a complex karyotype with four or more numerical or structural aberrations, -5 , deletion $(5 q)$, and -7 , whether alone or in combination with intermediate risk or other adverse risk abnormalities.

\subsection{Statistical analysis}

The overall survival (OS) for all patients was defined as the interval from the date of diagnosis to that of death. We applied the Kaplan-Meier method to estimate OS and 
Table 1 Patient characteristics

\begin{tabular}{ll}
\hline Age $($ year $)$ & $45(15-66)$ \\
Male/female & $390 / 248$ \\
WBC count $\left(\times 10^{9} / 1\right)$ & $13.7(0.4-709)$ \\
Hemoglobin $(\mathrm{g} / \mathrm{dl})$ & $8.3(3.8-17.2)$ \\
Platelet count $\left(\times 10^{9} / 1\right)$ & $52(0-890)$ \\
Bone marrow blasts $(\%)$ & $56(6-99)$
\end{tabular}

Values are presented as the median (range)

$W B C$ white blood cell

5-year survival. We compared survival rates between groups using the log-rank test (Stat View J 5.0). Differences were examined by the Chi-square test using Excel software. All $P$-values are two-sided, and values $<0.05$ were considered significant.

\section{Results}

\subsection{Patient characteristics}

Of the 809 registered patients, 638 were consistent with the WHO classification. Data were incomplete for 10 of the 638 patients. Table 1 lists the characteristics of the patients. The median age of all 638 patients (390 males and 248 females) was 45 years (range 15-66 years). The median values of $\mathrm{WBC}$, hemoglobin $(\mathrm{Hb})$, platelets, and the ratio of blasts in the bone marrow were $13.7 \times 10^{9} / 1$, $8.3 \mathrm{~g} / \mathrm{dl}, 52.0 \times 10^{9} / \mathrm{l}$, and $56.0 \%$, respectively.

\subsection{FAB classification}

Table 2 shows the FAB classification of the 638 patients. Most were classified as M2 $(n=261 ; 40.9 \%)$, followed by M4 $(n=148 ; 23.2 \%)$, and M1 $(n=109 ; 17.1 \%)$ with M0, M4Eo, M5a, M5b, M6, M7, and acute leukemia of ambiguous lineage comprising the remainder in that order.

\subsection{WHO classification and clinical characteristics}

Table 3 shows the patients categorized according to the WHO classification. The first category of AML with recurrent genetic abnormalities accounted for 171 patients (26.8\%), $133(20.8 \%)$ were in the second category of AML with MLD, $331(51.9 \%)$ were in the fourth category of AML not otherwise categorized, and $3(0.5 \%)$ were categorized as having acute leukemia of ambiguous lineage. Most patients in the second category were identical to those with a de novo MLD phenotype. We found that 144 patients diagnosed with the MLD phenotype comprised 133 (92.4\%) in the second category, 10 (7.0\%) with 11q23 abnormalities,
Table 2 Number of patients according to the FAB classification

\begin{tabular}{llcr}
\hline Subtype & Description & $\begin{array}{l}\text { No. } \\
\text { patients }\end{array}$ & of $\%$ \\
\hline M0 & $\begin{array}{l}\text { Minimally differentiated acute myeloid } \\
\text { leukemia (AML) }\end{array}$ & 30 & 4.7 \\
M1 & AML without maturation & 109 & 17.1 \\
M2 & AML with maturation & 261 & 40.9 \\
M4 & Acute myelomonocytic leukemia & 148 & 23.2 \\
& $\quad$ (AMMoL) & & \\
M4Eo & AMMoL with eosinophils & 23 & 3.6 \\
M5a & Acute monoblastic leukemia & 19 & 3.0 \\
M5b & Acute monocytic leukemia & 24 & 3.8 \\
M6 & Acute erythroleukemia & 16 & 2.5 \\
M7 & Acute megakaryoblastic leukemia & 5 & 0.8 \\
Acute leukemia of ambiguous lineage & 3 & 0.5 \\
Total & & 638 & 100 \\
\hline
\end{tabular}

Table 3 Number of patients according to the WHO classification

\begin{tabular}{|c|c|c|}
\hline Category and subtype & $\begin{array}{l}\text { No. of } \\
\text { patients }\end{array}$ & $\%$ \\
\hline I. AML with recurrent genetic abnormalities & 171 & 26.8 \\
\hline$t(8 ; 21)(\mathrm{q} 22 ; \mathrm{q} 22) ;(\mathrm{AML} 1 / \mathrm{ETO})$ & 113 & 17.7 \\
\hline $\begin{array}{l}\text { inv(16)(p13;q22) or } t(16 ; 16)(\mathrm{p} 13 ; \mathrm{q} 22) ;(\mathrm{CBF} \beta / \\
\text { MYH11) }\end{array}$ & 26 & 4.1 \\
\hline$t(15 ; 17)(\mathrm{q} 22 ; \mathrm{q} 12)(\mathrm{PML} / \mathrm{RAR} \alpha)$ & - & - \\
\hline 11q23(MLL)abnormalities & 32 & 5.0 \\
\hline II. AML with multilineage dysplasia & 133 & 20.8 \\
\hline Following MDS & - & - \\
\hline Without antecedent MDS & 133 & 20.8 \\
\hline III. AML and MDS, therapy-related & - & - \\
\hline Alkylating agent-related & - & - \\
\hline Topoisomerase type II inhibitor-related & - & - \\
\hline Other types & - & - \\
\hline IV. AML not otherwise categorized & 331 & 51.9 \\
\hline AML, minimally differentiated & 25 & 3.9 \\
\hline AML without maturation & 99 & 15.5 \\
\hline AML with maturation & 108 & 16.9 \\
\hline Acute myelomonocytic leukemia (AMMoL) & 63 & 9.9 \\
\hline AMMoL with eosinophilia & 5 & 0.8 \\
\hline Acute monoblastic leukemia & 8 & 1.3 \\
\hline Acute monocytic leukemia & 16 & 2.5 \\
\hline Acute erythroid leukemia & 6 & 0.9 \\
\hline Acute megakaryoblastic leukemia & 1 & 0.2 \\
\hline Acute leukemia of ambiguous lineage & 3 & 0.5 \\
\hline Total & 638 & 100 \\
\hline
\end{tabular}

and $1(0.7 \%)$ with acute leukemia of ambiguous lineage. Figure 1 shows the OS of each category. The 5-year survival rates of the first, second, and fourth categories were $58.2,22.5$, and $40.9 \%(P<0.0001)$, respectively. 


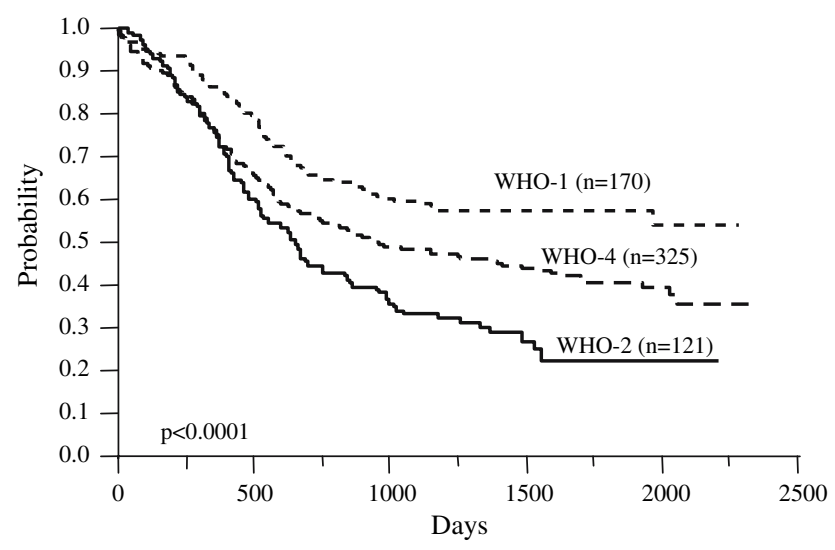

Fig. 1 Overall survival of patients categorized according to the WHO classification

Table 4 compares the clinical features among the WHO categories. The mean values of platelets, $\mathrm{WBC}, \mathrm{Hb}$, and the ratio $(\%)$ of blasts in bone marrow and of MPO-positive blasts significantly differed, whereas age did not significantly differ. Patients in the second category had a higher platelet count $\left(111.0 \times 10^{9} / 1\right)$, whereas those with $11 \mathrm{q} 23$ abnormalities had a lower count $\left(38.3 \times 10^{9} / 1\right)$ compared with those of other subtypes.

The WBC count of patients with $t(8 ; 21)$ was $1.4 \times 10^{9} / 1$ and lower than in other subtypes. The MPO-positive rate of blasts among patients with $t(8 ; 21)$ was higher $(93.3 \%)$ and that of patients in the second category was lower (34.0\%), than in other subtypes. All patients were grouped as highor low-MPO according to $\geq 50 \%$ or $<50 \%$ of MPO-positive blasts, respectively. A total of 339 patients $(53.1 \%)$ were classified as high-MPO, $268(42.0 \%)$ as low-MPO, and the MPO status of blasts could not be assessed in 31 (4.9\%). Figure 2 shows the OS of patients with high- or low-MPO. The 5-year survival rate for patients with high or low-MPO was 50.7 and $29.6 \%$, respectively $(P<0.0001)$.

\subsection{Cytogenetics}

All 638 patients were classified into favorable $(n=139$; $21.8 \%)$, intermediate $(n=413 ; 64.7 \%)$, and adverse $(n=54 ; 8.5 \%)$ cytogenetic risk groups (Table 5). Figure 3 shows the OS according to this stratification. The 5-year survival rates were $63.4,39.3$, and $0.0 \%$ in the favorable, intermediate (except for those with 11q23 abnormalities), and adverse risk groups, respectively, and $35.5 \%$ in the group with 11q23 abnormalities $(P<0.0001)$.

The numbers of patients with or without MLD and highor low-MPO in each cytogenetic risk group are listed in Table 6. None of those with the MLD phenotype were classified into the favorable risk group, while $129(89.6 \%)$ and $15(10.4 \%)$ of 144 patients with MLD were classified into intermediate or adverse risk groups, respectively. Only 15 patients $(4.4 \%)$ in the high-MPO group were classified as having an adverse risk, while $11(4.1 \%)$ in the low-MPO group were included in the favorable risk group.

The 32 patients with 11q23 abnormalities comprised 11 (34.4\%) with $t(11 ; 19), 9(28.1 \%)$ with $t(9 ; 11), 5(15.6 \%)$ with del(11)(q23), $4(12.5 \%)$ with $t(6 ; 11)$, and $3(9.4 \%)$ with $t(11 ; 17)$. Figure 4 shows the OS of the intermediate risk group. The 5-year survival rate was $44.0 \%$ in patients with a normal karyotype, $35.5 \%$ in those with $11 \mathrm{q} 23$ abnormalities, and $30.6 \%$ in other patients including those with $t(7 ; 11), t(6 ; 9)$, and $\mathrm{Ph}(+)$ abnormalities, respectively $(P=0.033)$.

Table 7 shows the relationship between $t(9 ; 11)(n=9)$ and other 11q23 abnormalities $(n=23)$. More patients with low-MPO, without MLD, or with the FAB M5 subtype were found in the group with $t(9 ; 11)$ than with other 11q23 abnormalities. The survival rates between the two groups did not significantly differ $(P=0.22$, data not shown).

\section{Discussion}

We attempted to classify selected patients who were reviewed morphologically and had available chromosomal data according to the WHO system. However, our series had some limitations in terms of analysis and patient selection. Although we obtained chromosomal data, genetic data were not available. Patients who were diagnosed with AML M3 or who had $t(15 ; 17)$, a history of MDS, or preceding hematological abnormalities, or who had previously undergone chemotherapy, were not eligible for the present study. However, multicenter trials might have some advantages in diagnosing AML according to the WHO classification, because morphological diagnoses and karyotypes are reviewed by the corresponding institutional committees.

The incidence of each category of the WHO classification was similar to those in several reports when patients with $t(15 ; 17)$ and therapy-related AML were excluded [2022]. We and several others have shown that approximately $30 \%$ of patients have recurrent genetic abnormalities. Multiplex reverse transcriptase-polymerase chain reaction (RT-PCR) assays have recently been applied to analyze cytogenetic abnormalities [21, 23, 24]. This method might cause the frequency of the first WHO category to increase. Thus, the multiplex RT-PCR assay might have to be incorporated into the WHO system. The JALSG has started a cohort study in which all AML patients in participating hospitals are registered and analyzed according to the WHO classification. That study should clarify the real ratios of the AML subtypes in the WHO classification. 

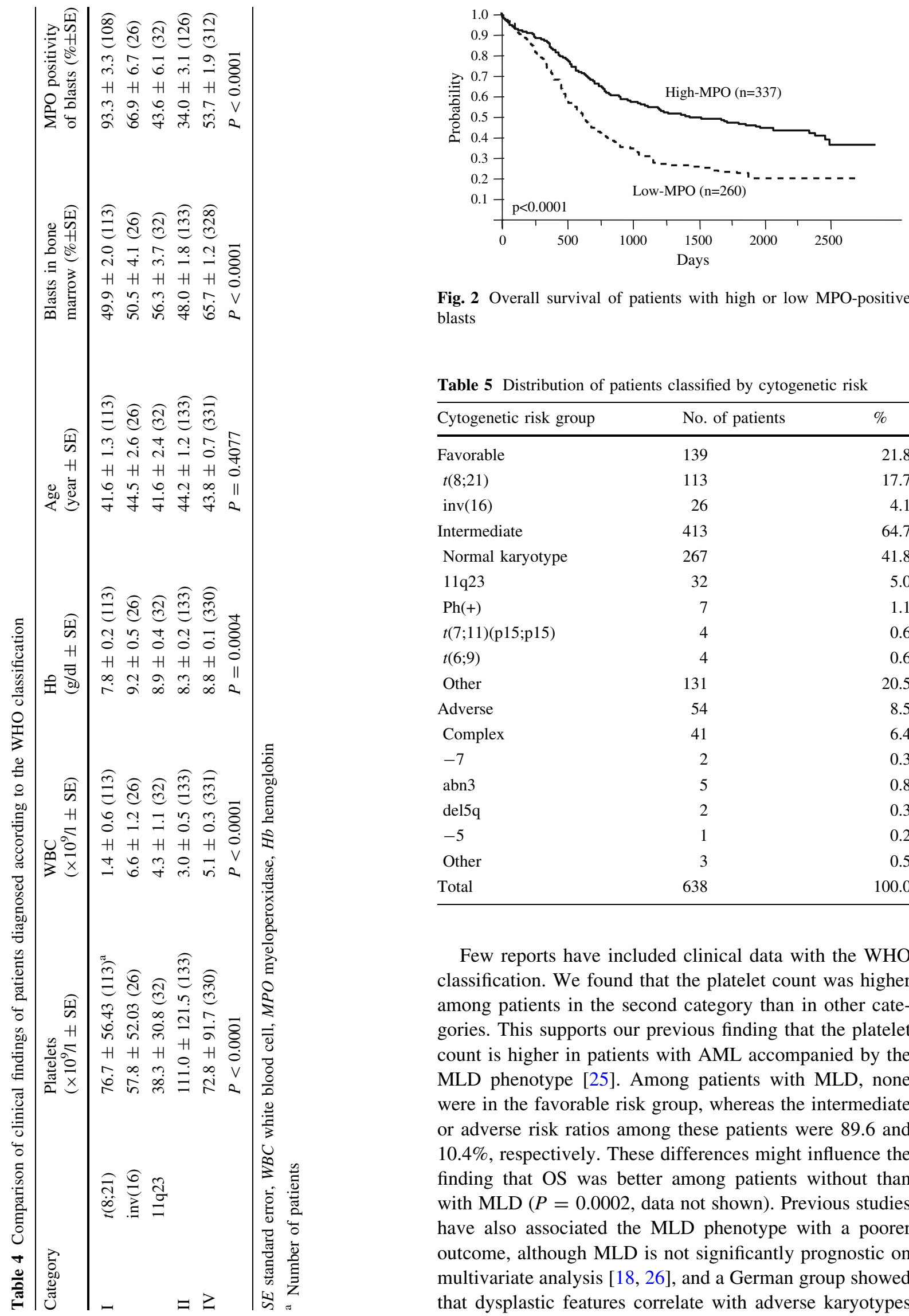

Fig. 2 Overall survival of patients with high or low MPO-positive blasts

Table 5 Distribution of patients classified by cytogenetic risk

\begin{tabular}{lrr}
\hline Cytogenetic risk group & No. of patients & $\%$ \\
\hline Favorable & 139 & 21.8 \\
$t(8 ; 21)$ & 113 & 17.7 \\
inv(16) & 26 & 4.1 \\
Intermediate & 413 & 64.7 \\
Normal karyotype & 267 & 41.8 \\
$11 \mathrm{q} 23$ & 32 & 5.0 \\
Ph(+) & 7 & 1.1 \\
$t(7 ; 11)(\mathrm{p} 15 ; \mathrm{p} 15)$ & 4 & 0.6 \\
$t(6 ; 9)$ & 4 & 0.6 \\
Other & 131 & 20.5 \\
Adverse & 54 & 8.5 \\
Complex & 41 & 6.4 \\
-7 & 2 & 0.3 \\
abn3 & 5 & 0.8 \\
del5q & 2 & 0.3 \\
-5 & 1 & 0.2 \\
Other & 3 & 0.5 \\
Total & 638 & 100.0 \\
\hline
\end{tabular}

Few reports have included clinical data with the WHO classification. We found that the platelet count was higher among patients in the second category than in other categories. This supports our previous finding that the platelet count is higher in patients with AML accompanied by the MLD phenotype [25]. Among patients with MLD, none were in the favorable risk group, whereas the intermediate or adverse risk ratios among these patients were 89.6 and $10.4 \%$, respectively. These differences might influence the finding that OS was better among patients without than with $\operatorname{MLD}(P=0.0002$, data not shown). Previous studies have also associated the MLD phenotype with a poorer outcome, although MLD is not significantly prognostic on multivariate analysis $[18,26]$, and a German group showed that dysplastic features correlate with adverse karyotypes 


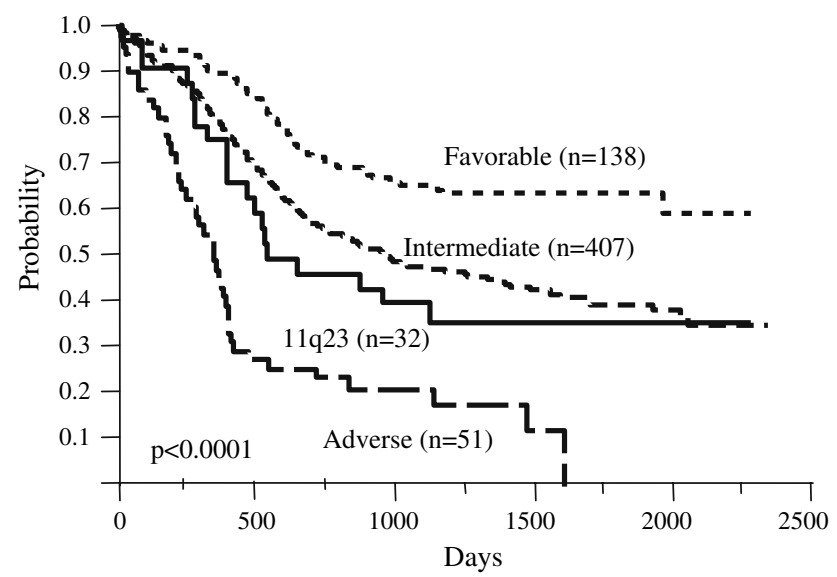

Fig. 3 Overall survival of patients stratified according to cytogenetic risk groups. Significant differences were observed between patients with a favorable, intermediate (except 11q23), and adverse karyotype $(P<0.0001)$

Table 6 Relationship between cytogenetic risk groups and MLD phenotype or MPO-positive rates of blasts

\begin{tabular}{llllr}
\hline & $\begin{array}{l}\text { Favorable } \\
n=139\end{array}$ & $\begin{array}{l}\text { Intermediate } \\
n=445\end{array}$ & $\begin{array}{l}\text { Adverse } \\
n=54\end{array}$ & Total \\
\hline MLD & & & & \\
+ & 0 & $129(89.5 \%)$ & $15(10.4 \%)$ & 144 \\
- & $138(28.2 \%)$ & $292(59.6 \%)$ & $38(7.8 \%)$ & 490 \\
Unknown & 1 & 2 & 1 & 4 \\
MPO & & & & \\
High & $123(36.3 \%)$ & $201(59.3 \%)$ & $15(4.4 \%)$ & 339 \\
Low & $11(4.1 \%)$ & $221(82.5 \%)$ & $36(13.4 \%)$ & 268 \\
Unknown & 5 & 23 & 3 & 31 \\
\hline
\end{tabular}

High- and low-MPO indicates a percentage of myeloperoxidase positive blasts $\geq 50$ or $<50 \%$, respectively

$M L D$ multilineage dysplasia

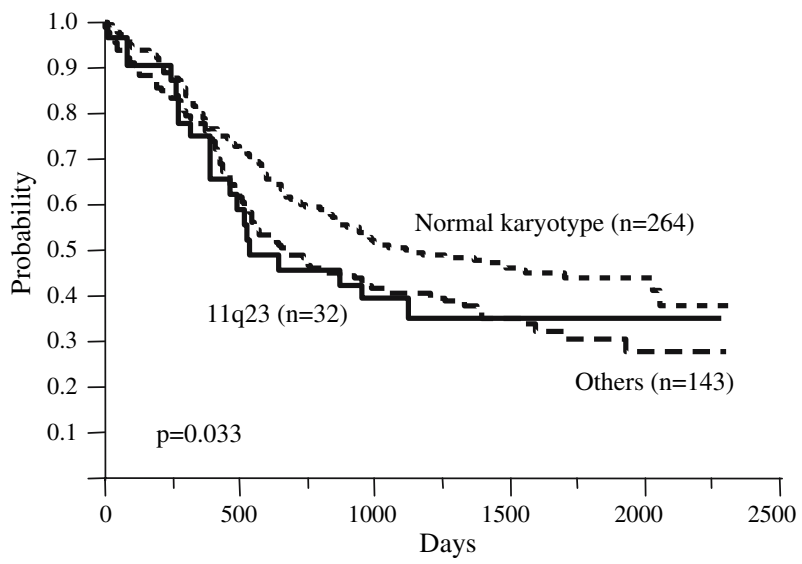

Fig. 4 Overall survival of patients with subtypes of intermediate cytogenetic risk. Significant differences were observed between patients with a normal karyotype and those with 11q23 abnormalities $(P=0.033)$
[26]. Furthermore, patients in the second category had a lower MPO-positive rate of blasts, whereas those with $t(8 ; 21)$ had a higher rate. Patients with high- and low-MPO were more frequently observed in the favorable and adverse risk groups, respectively. Multivariate analysis has shown that MPO is a significant factor affecting OS [19]. We did not assess prognostic factors by multivariate analysis here because the main theme of this study was to categorize patients according to the WHO classification, and we have already examined these in a previous series $[18,19]$.

Several studies have demonstrated the impact of specific cytogenetic abnormalities on survival in AML [3, 7-12, 20-22]. The cytogenetic risk groups stratified the AML patients in the present study according to the MRC system, as in these reports [3]. Therefore, we confirmed the clinical usefulness of cytogenetics as the first category of the WHO classification. We found that 32 patients had 11q23 abnormalities. The MRC system revealed that de novo and secondary AML patients with 11q23 abnormalities had an intermediate outcome with an OS rate of $45 \%$ at 5 years $(n=60$; median age, 17 years) in a younger cohort [3] and an OS rate of $0 \%$ at 5 years ( $n=11$; median age 64 years) in an elderly cohort [7]. In contrast, SWOG/ECOG trials including adult de novo AML patients (age, 16-55 years) assigned those with $11 \mathrm{q}$ abnormalities to the unfavorable cytogenetic subgroup [8]. Our data showed that patients with 11q23 abnormalities have an intermediate rather than adverse outcome. The prognostic effect of 11q23 abnormalities might depend on the partner gene. Several studies have shown that $11 \mathrm{q} 23$ abnormalities with $t(6 ; 11)$ and $t(10 ; 11)$ are associated with a poor prognosis, whereas $t(9 ; 11)$ is associated with a superior OS and such patients might respond well to intensive treatment, especially when the chemotherapy regimen includes high-dose cytarabine $[15,27-30]$. The CALGB study has shown that the median OS of 13.2 months among 23 patients with $t(9 ; 11)$ was significantly longer than the 7.7 months among 24 patients with other $11 \mathrm{q} 23$ rearrangements $(P=0.009)$ [30]. In a recent CALGB series of 54 patients with 11q23 abnormalities, 27 patients with $t(9 ; 11)$ had an intermediate outcome and a median OS of 13.2 months, whereas those with $t(6 ; 11)$ or $t(11 ; 19)$ had a poor outcome of 7.2 or 8.4 months [15]. Conversely, Schoch et al. showed that 14 patients with $t(9 ; 11)$ had a median OS of 10.0 months compared with the 12.8 months of 26 patients with other MLL rearrangements, and that the two cytogenetic groups did not significantly differ [13]. Our data showed that nine patients with $t(9 ; 11)$ were more frequently involved in M5. The MPO and MLD features significantly differed between patients with $t(9 ; 11)$ and those with other $11 \mathrm{q} 23$ abnormalities. However, the CALGB study found no significant differences in myelodysplastic features between the two 
Table 7 Comparison of $t(9 ; 11)$ and other 11q23 abnormalities

\begin{tabular}{|c|c|c|c|c|c|c|c|c|c|c|c|c|c|c|c|}
\hline & \multirow{2}{*}{ No. of patients } & \multicolumn{2}{|c|}{ Auer } & \multicolumn{2}{|c|}{ MPO* } & \multicolumn{2}{|c|}{ MLD* } & \multicolumn{6}{|c|}{ FAB } & \multirow{2}{*}{$\begin{array}{l}\text { Median age } \\
\text { (year) }\end{array}$} & \multirow{2}{*}{$\begin{array}{l}\text { Median survival } \\
\text { (day) }\end{array}$} \\
\hline & & + & - & High & Low & + & - & M1 & M2 & M4 & M4Eo & M5a** & M5b & & \\
\hline$t(9 ; 11)$ & 9 & 0 & 9 & 1 & 8 & 0 & 9 & 0 & 0 & 3 & 0 & 6 & 0 & 39 & 1031.00 \\
\hline Other $11 \mathrm{q} 23$ & 23 & 5 & 18 & 13 & 10 & 10 & 13 & 1 & 3 & 13 & 1 & 2 & 3 & 48 & 520.00 \\
\hline Total & 32 & 5 & 27 & 14 & 18 & 10 & 22 & 1 & 3 & 16 & 1 & 8 & 3 & 44.5 & 531.5 \\
\hline
\end{tabular}

High- and low-MPO indicates a percentage of myeloperoxidase-positive blasts $\geq 50$ or $<50 \%$, respectively

$M L D$ multilineage dysplasia

$* P<0.05, * * P<0.01$

cytogenetic groups [30]. In terms of OS, our results showed no significant differences between patients with $t(9 ; 11)$ and those with other 11q23 abnormalities $(P=0.22)$. Some problems are associated with the analyses of $11 \mathrm{q} 23$ abnormalities. We had few patients with these abnormalities, particularly individual translocations, and genetic analysis was not performed. Thus, the prognostic risk of 11 q23 abnormalities cannot be concluded from the present study. Nonetheless, these abnormalities were never associated with a favorable risk. To classify 11q23 abnormalities into each prognostic risk group, further investigations and genetic analyses of a large number of patients with 11q23 abnormalities are required.

The fourth WHO category, which is not otherwise categorized, accounted for $52 \%$ of patients in the present study. Most of them were classified into the intermediate risk group, and no prognostic subdivisions were valuable. Using cytogenetic features as a prognostic factor in groups with a normal karyotype has limitations, and such patients accounted for $64.6 \%$ of the intermediate risk group (data not shown). Additional factors are required to stratify these patients. We and several others suggested that differences could be based on molecular genetic analysis [22, 31-35]. For example, FLT3 mutations are important biomarkers of a normal karyotype and might be valuable for stratifying the intermediate risk group. Further follow-up studies might also shed light on the roles of FLT3 ITD mutations in the development of AML and aid their use as novel molecular targeting agents against AML [22, 32]. Bienz et al. identified CEBPA mutations, FLT3-ITD, and differing levels of BAALC expression as having independent prognostic significance in patients with a normal karyotype [33]. If these genetic markers can be confirmed as being of clinical significance, genetic analyses will probably be incorporated into the WHO classification.

In summary, our results confirmed those of previous studies showing the prognostic significance of cytogenetics, MLD, and MPO-positivity of blasts in AML. Furthermore, we categorized patients with de novo AML according to the WHO classification and showed the clinical characteristics and OS of each category.
Acknowledgments We thank the clinicians and leaders of all the institutions who entered their patients into the JALSG AML-97 study and provided the necessary data to make this study possible. This work was supported in part by a grant from the Ministry of Education, Culture, Sports, Science and Technology of Japan.

\section{References}

1. Bennett JM, Catovsky D, Daniel MT, et al. Proposals for the classification of acute leukaemias. French-American-British (FAB) co-operative group. Br J Haematol. 1976;33:451-8.

2. Bennett JM, Catovsky D, Daniel MT, et al. Proposed revised criteria for the classification of acute myeloid leukemia: a report of the French-American-British Cooperative Group. Ann Intern Med. 1985;103:620-5.

3. Grimwade D, Walker H, Oliver F, et al. The importance of diagnostic cytogenetics on outcome in AML: analysis of 1,612 patients entered into the MRC AML 10 trial. Blood. 1998;92:2322-33.

4. Harris NL, Jaffe ES, Diebold J, et al. World Health Organization classification of neoplastic diseases of the hematopoietic and lymphoid tissues: report of the Clinical Advisory Committee meeting-Airlie House, Virginia, November 1997. J Clin Oncol. 1999;17:3835-49.

5. Vardiman JW, Harris NL, Brunning RD. The World Health Organization (WHO) classification of the myeloid neoplasms. Blood. 2002;100:2292-302.

6. Brunning RD, Matutes E, Harris NL, et al. Acute myeloid leukaemias. In: Jaffe ES, Harris NL, Stein H, Vardiman JW (eds) Pathology and genetics of tumors of haematopoietic and lymphoid tissues. Lyon: IARC Press; 2001. p. 75-107.

7. Grimwade D, Walker H, Harrison G, et al. The predictive value of hierarchical cytogenetic classification in older adults with acute myeloid leukemia (AML): analysis of 1065 patients entered into the United Kingdom Medical Research Council AML 11 trial. Blood. 2001;98:1312-20.

8. Slovak ML, Kopecky KJ, Cassileth PA, et al. Karyotypic analysis predicts outcome of preremission and postremission therapy in adult acute myeloid leukemia: a Southwest Oncology Group/ Eastern Cooperative Oncology Group study. Blood. 2000;96:4075-83.

9. Rowe JM, Liesveld JL. Treatment and prognostic factors in acute myeloid leukaemia. Baillieres Clin Haematol. 1996;9:87-105.

10. Schoch C, Kern W, Schnittger S, Buchner T, Hiddemann W, Haferlach T. The influence of age on prognosis of de novo acute myeloid leukemia differs according to cytogenetic subgroups. Haematologica. 2004;89:1082-90.

11. Arber DA, Stein AS, Carter NH, Ikle D, Forman SJ, Slovak ML. Prognostic impact of acute myeloid leukemia classification: 
importance of detection of recurring cytogenetic abnormalities and multilineage dysplasia on survival. Am J Clin Pathol. 2003;119:672-80.

12. Bloomfield CD, Shuma C, Regal L, et al. Long-term survival of patients with acute myeloid leukemia: a third follow-up of the Fourth International Workshop on Chromosomes in Leukemia. Cancer. 1997;80:2191-8.

13. Schoch C, Schnittger S, Klaus M, Kern W, Hiddemann W, Haferlach T. AML with 11q23/MLL abnormalities as defined by the WHO classification: incidence, partner chromosomes, FAB subtype, age distribution, and prognostic impact in an unselected series of 1897 cytogenetically analyzed AML cases. Blood. 2003;102:2395-402.

14. Dohner K, Tobis K, Ulrich R, et al. Prognostic significance of partial tandem duplications of the MLL gene in adult patients 16 to 60 years old with acute myeloid leukemia and normal cytogenetics: a study of the Acute Myeloid Leukemia Study Group Ulm. J Clin Oncol. 2002;20:3254-61.

15. Byrd JC, Mrozek K, Dodge RK, et al. Pretreatment cytogenetic abnormalities are predictive of induction success, cumulative incidence of relapse, and overall survival in adult patients with de novo acute myeloid leukemia: results from Cancer and Leukemia Group B (CALGB 8461). Blood. 2002;100:4325-36.

16. Mitterbauer-Hohendanner G, Mannhalter C. The biological and clinical significance of MLL abnormalities in haematological malignancies. Eur J Clin Invest. 2004;34:12-24.

17. Miyawaki S, Sakamaki H, Ohtake S, et al. A randomized, postremission comparison of four courses of standard-dose consolidation therapy without maintenance therapy versus three courses of standard-dose consolidation with maintenance therapy in adults with acute myeloid leukemia: the Japan Adult Leukemia Study Group AML97 study. Cancer. 2005;104:2726-34.

18. Miyazaki Y, Kuriyama K, Miyawaki S, et al. Cytogenetic heterogeneity of acute myeloid leukemia (AML) with trilineage dysplasia: Japan Adult Leukemia Study Group-AML 92 study. Br J Haematol. 2003;120:56-62.

19. Matsuo T, Kuriyama K, Miyazaki Y, et al. The percentage of myeloperoxidase-positive blast cells is a strong independent prognostic factor in acute myeloid leukemia, even in the patients with normal karyotype. Leukemia. 2003;17:1538-43.

20. Arber DA, Stein AS, Carter NH, Ilie D, Forman SJ, Slovak ML. Prognostic impact of acute myeloid leukemia classification. Am J Clin Pathol. 2003;119:672-80.

21. Yanada M, Suzuki M, Kawashima K, et al. Long-term outcomes for unselected patients with acute myeloid leukemia categorized according to the World health Organization classification: a single-center experience. Eur J Haematol. 2005;74:418-23.

22. Bao L, Wang X, Ryder J, et al. Prospective study of 174 de novo acute myelogenous leukemias according to the WHO classification: subtypes, cytogenetic features and FLT3 mutations. Eur J Haematol. 2006;77:35-45.
23. Mayer-Monard S, Parlier J, Passweg J, et al. Combination of broad molecular screening and cytogenetic analysis for genetic risk assignment and diagnosis in patients with acute leukemia. Leukemia. 2006;20:247-53.

24. Olesen LH, Clausen N, Dimitrijevic A, Kerndrup G, Kjeldsen E, Hokland P. Prospective application of a multiplex reverse transcription-polymerase chain reaction assay for the detection of balanced translocations in leukaemia: a single-laboratory study of 390 paediatric and adult patients. Br J Haematol. 2004;127:5966.

25. Kuriyama K, Tomonaga M, Matsuo T, et al. Poor response to intensive chemotherapy in de novo acute myeloid leukaemia with trilineage myelodysplasia. Br J Haematol. 1994;86:767-73.

26. Haferlach T, Schoch C, Loffler H, et al. Morphologic dysplasia in de novo acute myeloid leukemia (AML) is related to unfavorable cytogenetics but has no independent prognostic relevance under the conditions of intensive induction therapy: results of a multiparameter analysis from the German AML Cooperative Group studies. J Clin Oncol. 2003;21:256-65.

27. Martineau M, Berger R, Lillington DM, Moorman AV, SeckerWalker LM. The $\mathrm{t}(6 ; 11)(\mathrm{q} 27 ; \mathrm{q} 23)$ translocation in acute leukemia: a laboratory and clinical study of 30 cases. Leukemia. 1998;12:788-91.

28. Dreyling MH, Schrader K, Fonatsch C, et al. MLL and CALM are fused to AF10 in morphologically distinct subsets of acute leukemia with translocation $\mathrm{t}(10 ; 11)$ : both rearrangements are associated with a poor prognosis. Blood. 1998;91:4662-7.

29. Scholl C, Schlenk RF, Eiwen K, Dohner H, Frohlin S, Dohner K. The prognostic value of MLL-AF9 detection in patients with $\mathrm{t}(9 ; 11)(\mathrm{p} 22 ; \mathrm{q} 23)$-positive acute myeloid leukemia. Haematologica. 2005;90:1626-34.

30. Mrozek K, Heinonen K, Lawrence D, et al. Adult patients with de novo acute myeloid leukemia and $\mathrm{t}(9 ; 11)(\mathrm{p} 22 ; \mathrm{q} 23)$ have a superior outcome to patients with other translocations involving band 11q23: a Cancer and Leukemia Group B study. Blood. 1997;90:4532-8.

31. Pasqualucci L, Liso A, Martelli PM, et al. Mutated nucleophosmin detects clonal multilineage involvement in acute myeloid leukemia: impact on WHO classification. Blood. 2006;108:4146-55.

32. Choudhary C, Schwable J, Brandts C, et al. AML-associated Flt3 kinase domain mutations show signal translocation differences compared with Flt3 ITD mutations. Blood. 2005;106:265-73.

33. Bienz M, Ludwig M, Mueller BU, et al. Risk assessment in patients with acute myeloid leukemia and a normal karyotype. Clin Cancer Res. 2005;11:1416-24.

34. Suzuki T, Kiyoi H, Ozeki K, et al. Clinical characteristics and prognostic implications of NPM1 mutations in acute myeloid leukemia. Blood. 2005;106:2854-61.

35. Ozeki K, Kiyoi H, Hirose $\mathrm{Y}$, et al. Biologic and clinical significance of the FLT3 transcript level in acute myeloid leukemia. Blood. 2004;103:1901-8. 